\title{
ESTRUCTURA GENÉTICA DE UN GRUPO DE CHIGÜIROS (Hydrochoerus hydrochaeris, Rodentia) EN LOS LLANOS ORIENTALES COLOMBIANOS
}

\author{
Luz Mery Bernal, ${ }^{94}$ Adriana Maldonado ${ }^{95}$ y \\ Gustavo Forero ${ }^{96}$
}

RESUMEN

Uno de los aspectos ecológicos clave para definir estrategias de manejo de poblaciones animales sociales es el tamaño y la estructura social del grupo. Esta investigación tuvo como objetivo identificar la estructura genética y variabilidad interna de un grupo de Chigüiros (Hydrochoerus hydrochaeris) que comparten el mismo espacio geográfico. La estructura genética fue evaluada con los estadísticos poblacionales básicos: número de alelos, frecuencias alélicas, tasas de polimorfismos y heterocigosis esperadas insesgadas a partir de marcadores microsatelitales. Obtuvimos amplificación positiva para cinco de los siete marcadores estudiados. De ello tuvimos una variación entre 20 y 31 individuos con perfiles alélicos confiables. El análisis de los perfiles alélicos muestra que existe una alta proporción de individuos heterocigotos en los marcadores Capy24, Capy 14 y Capy11, y sólo un marcador (Capy12), mostró alta proporción de ligamiento, este está en fase de análisis, al igual que las muestras genéticas; sin embargo, resultados previos dejan ver que existe una interesante variabilidad genética dentro del grupo.

Palabras clave: chigüiros, estructura genética, variabilidad genética.

\begin{abstract}
One of the key ecological aspects to define strategies of managing the animal social populations is the size and the social structure of the group. These investigations had as aim identify the genetic structure and intern variability of a group of Chigüiros (Hydrochoerus hydrochaeris) that share the same geographical space. Genetic structure was evaluated with the population basic statisticians: number of alleles, allele frequency, polymorphisms and heterozygosis. We obtained positive amplification for five of seven studied scoreboards. Of the obtained amplifications we had a variation between 20 and 31 individuals with profiles allelic reliable. The analysis of the profiles allelic shows that a high proportion of individuals exists heterocigotes in the scoreboards Capy24, Capy14 and Capy11, and only a scoreboard (Capy12), it showed high proportion of bond they are in phase of analysis, as the genetic
\end{abstract}

\footnotetext{
94 Lider Grupo Interdisciplinario de Estudios Sectoriales (GIES), Escuela de Ciencias Agrícolas Pecuarias y del Medio Ambiente, ECAPMA, UNAD.

95 Biologa y Química. MSc. Integrante Grupo GIES.

96 Licenciado Biología y Química. MSc. Integrante Grupo GIES
} 
samples; nevertheless, previous results led see that an interesting genetic variability exists inside the group.

Key words: Capybara, Genetic structure, Genetic variability

\section{INTRODUCCIÓN}

El sistema de apareamiento de una especie puede afectar las propiedades genéticas de la población (Wright, 1969) a través de la influencia de comportamientos sociales como la cooperación o la competencia entre individuos (Chesser, 1998). Las propiedades de los sistemas de apareamiento, a su vez, determinan la composición de los grupos y la variación genética dentro y entre las unidades sociales, es decir, la estructura genética del grupo (Ross, 2001). El chigüiro Hydrochoerus hydrochaeris, Lineaus 1766 es una especie social (Ojasti, 1980) que vive en agrupaciones familiares, compuestas por animales de ambos sexos, con predominancia de hembras (Herrera, 1999). Su sistema social muestra una jerarquía estrictamente dominada por los machos; dentro del grupo, sólo se admite un macho funcional (González-Jiménez, 1995), el cual puede acceder a varias hembras, mostrando un sistema de apareamiento poligínico. En este sentido, nuestra investigación está dirigida a responder el siguiente interrogante: ¿Cuál es la estructura genética de un grupo de chigüiros que comparten el mismo espacio geográfico?

\section{OBJETIVO GENERAL}

Determinar la estructura genética de una muestra de la población de chigüiros Hydrochoerus hydrochaeris de los Llanos Orientales, a través del uso de marcadores microsatelitales.

\section{OBJETIVOS ESPECÍFICOS}

Evaluar la efectividad de los microsatélites reportados por Herrera (2004), como herramienta para la caracterización de individuos en el análisis genético de poblaciones silvestres de chigüiros.

Determinar el grado de variabilidad genética en una muestra de individuos que hacen parte de una población de chigüiros de los llanos orientales.

Determinar la existencia de subgrupos o clusters de individuos relacionados genéticamente en una muestra de individuos que hacen parte de una población de chigüiros de los llanos orientales.

Proponer elementos que contribuyan en el planteamiento de estrategias de uso y aprovechamiento de las poblaciones de chigüiros en los llanos orientales.

\section{METODOLOGÍA}

Esta investigación de corte descriptivo constó de dos fases, una de campo y otra posterior de laboratorio. 


\section{Colecta de muestras}

Las muestras fueron tomadas en octubre de 2007 en el municipio de Hato, Corozal, Colombia. A cada uno de los individuos capturados se le tomó una muestra de sangre de la vena femoral. Las muestras fueron depositadas en tarjetas FTA $^{\circledR}$ (Whatman Bioscience, Cambridge, United Kingdom) y preservadas a temperatura ambiente.

\section{Procesamiento de muestras en laboratorio}

La extracción de ADN se realizó siguiendo el protocolo de Whatman. FTA ${ }^{\circledR}$ (2003). Posteriormente realizamos la amplificación del ADN para los microsatélites Capy7, Capy9, Capy11, Capy12, Capy14, Capy24, Capy25 y Capy26 reportados por Herrera (2004). En la amplificación usamos aproximadamente $20 \mathrm{ng}$ de $\mathrm{ADN}$ en un volumen total de reacción de $25 \mu \mathrm{l}$, con $0.3 \mu \mathrm{M}$ de cada cebador (primer), $0.3 \mathrm{mM}$ dNTP, 1 x Taq buffer (1.5 mM MgCl2, $10 \mathrm{mM}$ Tris- $\mathrm{HCl}, 50 \mathrm{mM} \mathrm{KCl})$ y 0.75 unidades de Taq polimerasa. La reacción se llevó a cabo en un termociclador PTC-100 25612 (MJR Research, Inc). Las temperaturas utilizadas fueron: $95^{\circ} \mathrm{C}$ durante 5 minutos; a continuación 34 ciclos de 30 segundos a $94{ }^{\circ} \mathrm{C}, 30$ segundos a la temperatura óptima de anillamiento $(54,58$, 60 y $61{ }^{\circ} \mathrm{C}$ dependiendo del marcador) y 45 segundos a $72{ }^{\circ} \mathrm{C}$. Finalmente, 5 minutos a $72{ }^{\circ} \mathrm{C}$. Los productos de la amplificación se almacenaron a $4{ }^{\circ} \mathrm{C}$. Corrimos geles de poliacrilamida al $6 \%$ en una cámara vertical de Secuencia ADN Thermo EC-160 con un tiempo de migración de 2 a 6 horas, dependiendo del tamaño del marcador, a $15 \mathrm{~W}$ constantes y a un voltaje que osciló entre los 300-400 V. Los geles se tiñeron con AgNO3.

\section{Análisis genético poblacional}

Para cada microsatélite estimamos los estadísticos genético-poblacionales básicos: número de alelos, frecuencias alélicas, tasas de polimorfismos y heterocigosis esperadas insesgadas, mediante el método de Nei (Ruiz-García 1991), a través de los programa GenePop, MiSat y Cervus. Adicionalmente, estimamos el posible equilibrio Hardy-Weinberg a través de tres métodos diferentes: la F de Wright (1965), la f de Robertson \& Hill (1984) y probabilidades exactas mediante el procedimiento de las cadenas de Markov (Raymond \& Rousset1995). Este procedimiento emplea la técnica de Cadenas de Markov Monte Carlo (MCMC) y permite utilizar los genotipos multi-locus para inferir estructura poblacional, mientras que los individuos son simultáneamente asignados a poblaciones específicas.

\section{RESULTADOS}

El DNA genómico fue extraído a partir de las 31 muestras de la especie en estudio, y la presencia de este fue verificada mediante electroforesis en geles de agarosa al $2 \%$ y evidenciados con tinción de SybrGreen. No se observaron bandas claras, razón por la cual procedimos a hacer una amplificación. 31 individuos amplificaron satisfactoriamente para alguno de los marcadores analizados. No se obtuvieron amplificaciones positivas para los marcadores Capy7 y Capy9, debido a que no se logró estandarizar el procedimiento de PCR. 
Capy11 obtuvo un total de 20 amplificaciones mientras que Capy14, Capy24 y Capy26 amplificaron en 30 muestras. Sólo logramos amplificar el total de muestras con Capy 12.

Diversidad Genética. El análisis de los perfiles alélicos muestra que existe una alta proporción de individuos heterocigotos en los marcadores Capy24, Capy14 y Capy11, y sólo un marcador (Capy12), mostró alta proporción de individuos homocigotos.

Los estadísticos de frecuencias alélicas calculados con el programa Cervus 3.03 - (C) Copyright Tristan Marshall 1998-2007 (Field Genetics Ltd - www.fieldgenetics.com), así como el posible desequilibrio de Ligamiento que indica si los marcadores estudiados se encontraban bajo presión de selección o en desequilibrio de ligamiento están en fase de análisis.

\section{REFERENCIAS BIBLIOGRÁFICAS}

ALDERSON, G.W., H.L. GibbS and S.G. Sealy, (1999), Determining the reproductive behaviour of individual brown-headed cowbirds using microsatellite DNA markers. Animal Behaviour 58, 895-905.

AlHO, C.J., Z.M. Campos \& H.C. Gonçalves. (1989), Ecology, social behavior and managment of the capybara (Hydrochaeris hydrochaeris) in the pantanal of Brazil. Advances in Neotrop. Mammal. 163-194.

BECKMAN, J., S.C. Banks, P. Sunnucks, A. Lill and A.C. Taylor, (2007), Phylogeography and environmental correlates of a cap on reproduction: teat number in a small marsupial, Antechinus agilis. Molecular Ecology 16, 1069-1083.

BERG, E.C., (2005), Parentage and reproductive success in the white-throated magpie-jay, Calocitta formosa, a cooperative breeder with female helpers. Animal Behaviour 70, $375-385$.

COLTMAN D.W., D.W. Bowen y J.M. Weith. (1996), PCR primers for harbour seal (Phoca vitulina concolour) microsatellites amplify polymorphic loci other pinniped species. Molecular Ecology 5,161-163. Chesser 1998.

DOBSON, F.S. y B. Zinner. (1998), Social groups, genetic structure, and conservation. En: Festa-Bianchet, M. Apoollonio, M. (Eds.) (2003). Animal Conservation and Wildlife Conservation. Island Press.

EGUiARTE, L. E., V. Souza y X. Aguirre (Compiladores). (2007), Ecología Molecular. Secretaría de Medio Ambiente y Recursos Naturales. Instituto Nacional de Ecología Universidad Nacional Autónoma de México. Comisión Nacional para el Conocimiento y Uso de la Biodiversidad. Mexico. 
ESCOBAR, A. y González Jiménez, E. 1976. Estudio de la competencia alimenticia de los herbívoros mayores del llano inundable con referencia especial al chigüire, Hydrochoerus hydrochaeris. Agronomía Tropical XXVI: 215-227.

FORERO, J. (1999), Dieta alimentaria del Chigüiro (Hydrochaeris hydrochaeris) en Caño Limón, Arauca, Colombia. Tesis para optar al título de Biólogo. Universidad de los Andes. Departamento de ciencias biológicas. Laboratorio de ecología vegetal. Santafé de Bogotá.

GONZÁLEZ-JIMÉNEZ, E. (1995), El capibara. Estado actual de su producción. Serie FAO de Producción y Sanidad animal.

HERRERA, E. (1999), Comportamiento, conservación y manejo de fauna Silvestre: el caso del capibara en Venezuela. Etología. 7: 41-46.

HERRERA, E. y D. Macdonald. (1989), Resource utilization and territoriality in group-living capybaras (Hydrochaeris hydrochaeris). J. Anim. Ecol. 58, 667-679.

LAME P. and P.J., Lagoda., (1996), Lagoda Microsatellites, from molecules to populations and back Trend Ecol Evol 11, 424-429.

MESA E. y J. Sánchez. (2002), Implementación de la fase inicial del programa de manejo del chigüiro (Hydrochaeris hydrochaeris) en semicautiverio, como estrategia de conservaciónde la biodiversidad en la orinoquía colombiana y acopio de información complementaria. Convenio de cooperación científica y tecnológica No. 085. Ministerio del Medio Ambiente - Universidad Nacional de Colombia. Bogotá.

OJASTI, J., (1973), Estudio biológico del chigüiro o capibara. Fondo Nacional de Investigaciones Agropecuarias, Caracas, Venezuela.

OJASTI, J., (1980), Ecology of capibara raising on inundated savannas of Venezuela. Tropical Ecology \& Development: 287-293.

OJASTI, J., (1991), Human Exploitation of Capybara. Cap. 17 (págs. 236-253) en: Neotropical Wildlife Use and Conservation (Robinson, J.G. y Redford, K.H., eds.). Chicago University Pres, Chicago, USA.

PARDO, B.G., M. Hermida, C. Fernandez, C. Bouza, M. Perez, A. Llavona, L. Sanchez y P. Martinez. (2006), A Set of Highly Polymorphic Microsatellites Useful for Kinship and Population Analysis in Turbot (Scophthalmus maximus L.) Aquaculture Research $37,1578-1582$. 
PEREA, J. y S. Ruiz. 1977. Organización social y hábitos territoriales del chigüiro. Tesis de pregrado Universidad Nacional de Colombia.

POLLOCK, D.D., (1998), Microsatellite Behavior with Range Constraints: Parameter Estimation and Improved Distances for Use in Phylogenetic Reconstruction. Theoretical Population Biology 53, 256-271.

RAYMOND M., F. Rousset. (1995), GENEPOP (version 1.2): population genetics software for exact tests and ecumenicism. Journal of Heredity 86: 248-249.

ROBERTSON, a., Hill, W. G. (1984), Deviations from Hardy-Weinberg prpportions, sampling variances and use in estimation of inbreeding coefficients. Genetics 107: 703718.

RUIZ-GARCÍA, M. (1991), Más sobre la Genética de Poblaciones de Felis catus en la costa Mediterránea española: un análisis de la estructura genética de las poblaciones naturales de gatos. Evol. Biol., 5: 227-283.

ROSS, K.G. (2001), Molecular Ecology of Social Behaviour: Analyses of Breeding Systems and Genetic Structure. Molecular Ecology 10(265-284).

SCANDURA, M. (2004), The Use of Microsatellites in the Study of Social Structure in Large Mammals: Italian Wolf and Fallow Deer as Case Studies. PhD Thesis. University of Bielefeld, Faculty of Biology.

SOINI, P. y M. Soini. (1992), Ecología del ronsoco o capibara (Hydrochaeris hydrochaeris) en la reserva nacional Pacaya-Samiria, Perú. Folia Amazónica 4, 119-133.

SUGG, D. W., R. K. Chesser, F. S. Dobson y J. L. Hoogland, 1996. Population Genetics meets Behavioral Ecology. Trends in Ecology \& Evolution 11, 338-342.

WHATMAN. FTA ${ }^{\circledR}$ protocols: collect transport, archive and access nucleic acids all at room temperature 2002; URL http:// www.cosmobio.com.ar/docs/fta\%20protocols.pdf.

WHATMAN. Room Temperature Sample Collection, Storage and Purification of Nucleic Acids 2003; URL http://www.touchbriefings.com/pdf/16/fdd031_t_whatman.pdf.

WRIGHT, S. 1965. The Interpretation of Population Structure by F-statistics with Special Regards to Systems of Mating. Evolution 19: 395-420. 